\title{
A PERCEPÇÃO DOS HÓSPEDES DE NEGÓCIOS QUANTO AO DESEMPENHO DA QUALIDADE DOS SERVIÇOS PRESTADOS NOS HOTÉIS DE FLORIANÓPOLIS: UMA ANÁLISE A PARTIR DO CONTEÚDO GERADO NO WEBSITE BOOKING.COM
}

PERCEPTIONS OF BUSINESS GUESTS OF THE QUALITY OF SERVICES PROVIDED IN HOTELS OF FLORIANÓPOLIS: AN ANALYSIS BASED ON CONTENT GENERATED ON WEBSITE BOOKING.COM

LA PERCEPCIÓN DE LOS HUÉSPEDES DE NEGOCIOS EN RELACIÓN AL DESEMPEÑO DE LA CALIDAD DE LOS SERVICIOS PRESTADOS EN LOS HOTELES DE FLORIANÓPOLIS: UN ANÁLISIS A PARTIR DEL CONTENIDO GENERADO EN EL WEBSITE BOOKING.COM

Tânia Regina Egert Petry Mestranda em Turismo e Hotelaria do Programa de Pós-Graduação Stricto Sensu Universidade do Vale do Itajaí - UNIVALI taniaegert@gmail.com

\section{Cibelli de Medeiros Pickler}

Mestre em Administração Universitária - UFSC belly_rms@yahoo.com.br

\section{Carlos Alberto Tomelin}

Docente do Programa de Pós-Graduação Stricto Sensu - Mestrado e Doutorado em Turismo e Hotelaria e Vice-Reitor de Planejamento e Desenvolvimento Institucional da Universidade do Vale do Itajaí - UNIVALI 


\begin{abstract}
RESUMO: A fim de melhorar sua participação nacional no segmento de turismo de negócios e eventos, os empreendedores da cidade de Florianópolis devem primar pela qualidade dos serviços oferecidos. Para tanto, com os consumidores cada vez mais exigentes e apoiados em sites de avaliações de serviços para auxiliar nas suas escolhas, uma maior atenção das empresas neste sentido denota-se necessária. No turismo, o chamado Conteúdo Gerado pelo Usuário (CGU) se populariza à medida que serve como fonte de informação. Por esta razão, o presente artigo teve como objetivo avaliar a percepção dos hóspedes de negócios quanto ao desempenho da qualidade dos serviços prestados em seis hotéis da cidade de Florianópolis. Para tal, utilizou-se um estudo do tipo exploratório-descritivo, tendo como método analítico o qualitativo. Foram avaliados 1.133 comentários postados pelos usuários no site de viagens Booking.com de acordo com as dimensões da qualidade do Modelo SERVPERF. A escolha do site se deu por sua penetração de escala junto ao público investigado. Os elementos de maior destaque apontados pelos hóspedes foram aferidos em escala de prioridade, utilizando-se para a análise de textos o Software NVIVO. Diante dos resultados, observou-se que os aspectos tangíveis tiveram maior grau de prioridade nas avaliações dos hóspedes, com destaque para a localização, o café da manhã e o conforto do quarto, como os principais pontos fortes, o que pode auxiliar os hotéis na formulação de estratégias para maximizar ainda mais estes aspectos. Em contrapartida, os equipamentos e as instalações foram mal avaliados, o que denota oportunidades de melhoria nas evidências físicas assinaladas. Ao se pensar nas categorias serviços e infraestrutura, percebe-se a inter-relação sobre atributos de qualidade nos aspectos tangíveis avaliados pelos hóspedes.
\end{abstract}

PALAVRAS-CHAVE: Qualidade do serviço. SERVPERF. Hotéis de Florianópolis.

ABSTRACT: In order to improve their participation in the business and events tourism market in Brazil, entrepreneurs in the city of Florianópolis should prioritize the quality of services offered. With increasingly demanding consumers, assisted in making their choices by websites that evaluate the services, companies need to pay more attention to the aspect of quality. In tourism, the so-called User Generated Content (UGC) has become popular, serving as a source of information. The aim of this article was to evaluate the perceptions of business guests in relation to the quality of the services provided in six hotels in the city of Florianópolis. For this purpose, an exploratory and descriptive study was carried out, using qualitative and analytical methods. A total of 1133 comments posted by users on Booking.com were used, according to the dimensions of SERVPERF quality Model. The choice of website was based on its reach among the public investigated. The most prominent elements indicated by guests were checked on a scale of priority, using the software NVIVO to analyze the texts. Based on the results, it was observed that the tangible aspects had higher priority in the reviews, especially the location, the breakfast and the comfort of the room, which were seen as the main strengths. Hotels should therefore formulate strategies to further maximize these aspects. In contrast, equipment and facilities were poorly evaluated, suggesting opportunities for improvement in the physical aspects indicated. Thinking of the categories services and infrastructure, an interrelation is seen in the attributes of quality, in the tangible aspects evaluated by the guests.

KEYWORDS: Quality of service. SERVPERF. Florianópolis Hotels.

RESUMEN: A fin de mejorar su participación nacional en el segmento de turismo de negocios y eventos, los emprendedores de la ciudad de Florianópolis deben priorizar la calidad de los servicios ofrecidos. Para ello, y con los consumidores cada vez más exigentes y apoyados en sitios 
de evaluación de servicios para auxiliarlos en sus elecciones, se muestra necesaria una mayor atención de las empresas en este sentido. En el turismo se populariza el denominado Contenido Generado por el Usuario (CGU), en la medida en que sirve como fuente de información. Por esta razón, el presente artículo tuvo como objetivo evaluar la percepción de los huéspedes de negocios en relación al desempeño de la calidad de los servicios prestados en seis hoteles de la ciudad de Florianópolis. Para eso se utilizó un estudio de tipo exploratorio descriptivo, que tenía como método analítico el cualitativo. Se evaluaron 1.133 comentarios publicados por los usuarios en el sitio de viajes Booking.com, de acuerdo con las dimensiones de la calidad del Modelo SERVPERF. La elección del sitio se justificó por su penetración de escala sobre el público investigado. Los elementos de mayor destaque apuntados por los huéspedes fueron estimados en escala de prioridad, utilizándose para el análisis de textos el Software NVIVO. Frente a los resultados, se observó que los aspectos tangibles tuvieron mayor grado de prioridad en las evaluaciones de los huéspedes, con destaque para la ubicación, el desayuno y la comodidad de la habitación, como los principales puntos fuertes, lo que puede auxiliar a los hoteles en la formulación de estrategias para maximizar aún más estos aspectos. En contrapartida, los equipamientos y las instalaciones fueron mal evaluados, lo que denota oportunidades de mejoría en las evidencias físicas señaladas. Al pensar en las categorías de servicios e infraestructura, se percibe la interrelación entre los atributos de calidad en los aspectos tangibles evaluados por los huéspedes.

PALABRAS CLAVE: Calidad del servicio. SERVPERF. Hoteles de Florianópolis.

INTRODUÇÃO

egundo a Pesquisa anual de conjuntura econômica do turismo, o
ano de 2015 deverá apresentar relativo crescimento nos negócios
relacionados ao setor, com saldo de $43 \%$ em comparação a 2014, levando-se em conta uma variação média anual de 4,1\%. Conforme levantamento realizado com empresários do setor, 64\% indicaram uma perspectiva otimista de seus negócios, $15 \%$ classificaram como uma fase de estabilidade e $21 \%$ demostraram uma visão de redução (MTUR, 2015).

Os hotéis são um dos principais segmentos responsáveis pela expectativa otimista dos empresários, visto que com a desvalorização do real frente ao dólar, espera-se que a vinda de turistas estrangeiros ao país possa contribuir para o aumento da taxa de ocupação dos meios de hospedagem. Além disso, outro motivo destacado como forte impulsionador desse mercado é a captação de negócios e eventos. Este segmento é considerado estratégico, devido à capacidade de minimizar os efeitos da sazonalidade e ocorrer independentemente da existência de atrativos naturais ou culturais. Nesse contexto, o Brasil figura na $10^{a}$ posição entre os países que mais receberam 
congressos e eventos internacionais no ano de 2014 (MTUR, 2015; MTUR, 2010; ABEOC, 2015).

No cenário nacional, a capital do Estado de Santa Catarina - Florianópolis - ocupa $\circ 6^{\circ}$ lugar entre as cidades brasileiras que mais receberam eventos (ABEOC, 2015). Dos meios de hospedagem utilizados pelos participantes, segundo pesquisa realizada pelo Convention \& Visitors Bureaux em parceria com a Fundação Getúlio Vargas, $87,20 \%$ fez uso dos serviços de hotéis da cidade, $10,90 \%$ ficou hospedado na casa de amigos ou parentes e $2 \%$ afirmou ter utilizado outros tipos de hospedagem (Florianópolis e região C\&VB; FGV, 2010). Estima-se, ainda, que no período de 2008 a fevereiro de 2015, a cidade tenha recebido mais de 250 mil pessoas por meio dos congressos realizados, o que contribuiu para a ocupação hoteleira da cidade (ABEOC, 2015).

Dessa forma, justifica-se a importância dos hotéis priorizarem esforços para atender aos atributos considerados mais significativos por essa demanda de clientes. Ademais, com o avanço da comunicação via internet, as empresas passaram a ser avaliadas via on-line pelos consumidores, por meio do Conteúdo Gerado pelo Usuário (CGU). Em portais de viagens como o Booking.com, os comentários dos hóspedes de hotéis tornaram-se úteis para a tomada de decisão por parte de novos usuários, que recorrem ao site para verificar o que já foi publicado sobre o empreendimento (YACOUEL; FLEISCHER, 2012).

Nesse sentido, analisar o conteúdo gerado pelos hóspedes por meio de sites de viagens caracteriza-se como uma maneira eficiente de descobrir o que pode ser mudado ou melhorado nos serviços prestados, especificamente na hotelaria. A este respeito, a Booking.com se destaca como uma das empresas líderes em informação relacionada à hospedagem no mundo, assim como no Brasil, fato que legitimou pela escolha desse site como objeto de estudo.

O objetivo desta pesquisa foi o de avaliar a percepção dos hóspedes de negócios quanto ao desempenho da qualidade dos serviços prestados em seis hotéis da cidade de Florianópolis, utilizando a escala SERVPERF de Cronin \& Taylor (1992).

Foram coletados todos os comentários publicados por hóspedes de negócios, com hospedagem nos hotéis da cidade, no período entre 13 de maio de 2014 
e 14 de julho de 2015. Na sequência, ao ser verificada a preponderância de comentários deste perfil de público em seis hotéis específicos, em detrimento de outros com menor número de avaliações, estes foram os escolhidos a participar da pesquisa. O estudo analisou os dados de forma geral, sem fazer comparações entre os hotéis. No que diz respeito à análise das informações coletadas, fez-se uso da escala SERVPERF, de Cronin \& Taylor (1992), para verificar as variações da qualidade em relação às cinco dimensões do modelo, quais sejam: tangibilidade, confiabilidade, capacidade de resposta, segurança e empatia.

Dessa maneira, identificou-se, a partir do conteúdo gerado pelos usuários (CGU) no site booking.com, os pontos fortes e fracos percebidos pelo consumidor ao utilizar os serviços dos hotéis pesquisados, permitindo aferir níveis de prioridade em cada dimensão analisada no modelo SERVPERF. O estudo visa demonstrar que o CGU consiste em uma rica fonte de informação, que os hotéis podem utilizar constantemente como forma de orientar suas estratégias para a melhoria contínua dos serviços e da infraestrutura oferecidos.

Por fim, a pergunta para a qual se buscou resposta nesta pesquisa foi: Como os hotéis da cidade de Florianópolis podem se utilizar do Conteúdo Gerado pelo Usuário (CGU) no site Booking.com como ferramenta para auxiliar suas estratégias de melhoria dos serviços oferecidos?

Este estudo caracteriza-se como qualitativo, descritivo-exploratório, baseado em revisão bibliográfica sobre assuntos como o conteúdo gerado pelo usuário (CGU) no Turismo e Hotelaria, a Qualidade dos Serviços na Hotelaria, além do Modelo de avaliação da qualidade dos serviços SERVPERF. A apresentação do trabalho está estruturada com a presente introdução, seguida pela revisão da literatura, metodologia, resultados e discussões e considerações finais.

\section{REVISÃO DA LITERATURA}

A revisão da literatura abordará conceitos relevantes para o estudo e que contribuíram no desenvolvimento da pesquisa e escolha da metodologia adotada, como: o conteúdo gerado pelo usuário (CGU) no Turismo e Hotelaria, a Qualidade dos Serviços na Hotelaria e o Modelo de avaliação da qualidade dos serviços (SERVPERF). 


\section{O CONTEÚDO GERADO PELO USUÁRIO (CGU) NO TURISMO E HOTELARIA}

Com o avanço das tecnologias da informação (TICs), os consumidores estão confiando cada vez mais nos serviços turísticos disponíveis na internet. Segundo dados divulgados pelo Ministério do Turismo, a porcentagem de turistas estrangeiros que utilizou a internet como fonte de informação para visitar o Brasil durante a Copa do mundo de futebol foi de 68\% (MTUR, 2014).

O relatório anual feito pela Phocuswright (2014) sobre o consumo de viagens nos EUA revelou que a porcentagem de viajantes americanos que reservam via on-line estabilizou no ano passado, ficando em 39\%, depois de aumentar 32\% em 2012. Enquanto isso, as reservas de viagens nos Estados Unidos feitas de forma off-line, como em agências de viagens e diretamente com fornecedores, mantiveram-se estáveis após anos de redução, à medida que subiram os gastos em viagens mais complexas e a procura por consultoria antes da viagem.

Os usuários acessam a internet antes de suas viagens para buscar informações que auxiliam no seu planejamento e depois da sua realização, para compartilhar experiências uns com os outros (SILVA; MENDES-FILHO, 2014). Essas informações on-line geradas pelos internautas são conhecidas como Conteúdo gerado pelo usuário (CGU), uma forma de divulgação boca a boca eletrônica que tem crescido nos últimos anos, acarretando o desenvolvimento de uma série de ferramentas genericamente definidas como mídias sociais on-line (MENDESFILHO; CARVALHO, 2014; SILVA; MENDES-FILHO, 2014). Outros autores, como Sartori e Reis (2010); e Quiroga, Mondo e de Castro Júnior (2014), tratam esse monitoramento das informações referente à percepção dos consumidores contidas na internet como Reputação On-line.

Neste sentido, verificam-se estudos recentes na área de turismo e hotelaria que fizeram uso do CGU como fonte de informação para suas pesquisas. Limberger, Anjos, Meira e Anjos (2014) investigaram a correlação entre a satisfação geral e os critérios de avaliação de hotéis de 03 a 05 estrelas, com base em informação obtida no site TripAdvisor. Os critérios de avaliação dos 
serviços que são disponibilizados neste portal são: satisfação global, valor (custo-benefício), localização, qualidade do sono, quartos, limpeza e serviço. Para encontrar a correlação entre satisfação global e outros atributos, o trabalho utiliza-se de análise multivariada (YAMAMOTO; ISHIDA \& OHTA, 2004).

Mendes-Filho e Carvalho (2014) analisaram os fatores que influenciam o uso do conteúdo gerado pelo usuário no planejamento de viagens. Neste estudo, a metodologia para a coleta de dados foi o processo de planejamento de uma viagem por meio da análise dos comentários de sites como o TripAdvisor e Booking.com, e suas influências sobre a intenção na escolha do hotel.

Já Corrêa e Hansen (2014) avaliaram a percepção da qualidade de serviços prestados por restaurantes da cidade de São Paulo. Para tanto, utilizaram como metodologia a abordagem empírica para análise do CGU por meio da escala SERVQUAL, buscando mensurar o gap que existe entre expectativa e percepção para definir a qualidade do serviço avaliado. Para a análise dos comentários foram divididos conforme as dimensões da escala SERVQUAL e de acordo com os comentários positivos e negativos.

No trabalho de Quiroga, Mondo e de Castro Júnior (2014), examinou-se a reputação on-line dos Meios de Hospedagem de Garopaba e Imbituba. Os pesquisadores empregaram como método a categorização dos comentários, por nome do meio de hospedagem; título do comentário do hóspede; o próprio comentário deixado; a data do comentário e data que ocorreu a hospedagem; a categoria, se viajou em família, sozinho, ou a negócios; e a qualidade do comentário, se negativo ou positivo.

Nessa mesma perspectiva, Gândara, Brea e Manosso (2013), estudaram a qualidade da experiência vivida por hóspedes de hotéis termais da Galícia Espanha. Foram coletados comentários postados nos sites Trivago, Tripadvisor e Booking, porém neste caso a análise dos pesquisadores ocorreu dentro das dimensões da experiência definidas por Pine II e Gilmore (1999), observandose a reputação on-line dos hotéis frente aos seus clientes.

A partir dos resultados obtidos, Limberger et al. (2014) apontaram para um maior índice de correlação da satisfação geral com os critérios quarto, serviço ofertado e custo-benefício. Assinalaram, sobretudo, que os gestores do setor 
devem compreender o conteúdo gerado pelos usuários, pois a hotelaria se apresenta como um dos segmentos mais expostos às mídias sociais.

Na pesquisa realizada por Mendes-Filho e Carvalho (2014), revelou-se que a maioria dos entrevistados foi influenciada pelo CGU na escolha do hotel (75\%). Contudo, segundo os dados obtidos, apenas $12 \%$ dos entrevistados fazem comentários on-line após a realização da sua viagem.

No estudo de Corrêa e Hansen (2014), foi constatado que os restaurantes avaliados no trabalho são considerados competentes, confiáveis, oferecem bons serviços de alimentação e atendem às necessidades dos consumidores.

Já na pesquisa de Quiroga, Mondo e de Castro Júnior (2014), verificou-se que a reputação on-line dos meios de hospedagem de Imbituba é melhor que a reputação on-line dos meios de hospedagem de Garopaba. O que confirma a existência de pontos a serem superados pelos empreendimentos, na busca pela satisfação do cliente deste destino turístico (MOENS; LI \& CHUA, 2014).

Como um dos principais resultados do trabalho de Gândara, Brea e Manosso (2013), observou-se que a maior parte dos hóspedes que ficou hospedada em hotéis termais da Galícia avaliou positivamente a qualidade da experiência vivida. Além disso, o estudo destaca a importância dos comentários postados on-line para realização das análises da pesquisa.

O uso do conteúdo gerado pelo usuário apresenta-se como uma ferramenta na direção do maior conhecimento sobre os clientes, oportunizando a melhoria na qualidade dos serviços prestados pelas empresas turísticas (FRIEDLANDER, 2012; MARCHIORI; CANTONI, 2011).

Sartori e Reis (2010) acrescentam que o gerenciamento da reputação on-line das empresas em sites de avaliações de produtos e serviços pode ser percebido como forma legítima de inteligência competitiva, tornando-se cada vez mais contundente e decisivo para os negócios corporativos. Conjuntamente ao monitoramento passivo dos comentários postados, a gestão do conteúdo online inclui, sobretudo, o posicionamento, a análise criteriosa e a tomada de decisões voltadas ao lançamento de novos produtos, processos e serviços.

Com isso, verifica-se relevante as empresas hoteleiras estarem atentas a 
sites de viagens a exemplo do Booking.com, Tripadvisor e Decolar, para fazer a gestão on-line do conteúdo publicado. No que tange à preocupação com a confiabilidade do conteúdo gerado, o site de viagens Booking.com possibilita a avaliação somente de hóspedes que reservaram por meio do portal, restringindo as avaliações a hóspedes "reais", ou seja, aqueles que efetivamente fizeram uso dos serviços de hospedagem (BOOKING, online, 2015).

No caso específico dos hotéis que atendem a turistas de negócios e eventos, devido à dinamicidade desse mercado, os hóspedes apresentam expectativas variadas. O turista de negócios vai desde o alto executivo até pequenos grupos de trabalho operacional. Por isso, faz-se necessário conhecê-los antes de tudo (FERREIRA, 2010).

Quiroga, Mondo e de Castro Júnior (2014) relatam que a verificação das opiniões on-line procura compreender os pontos fortes e fracos dos meios de hospedagem utilizados pelos hóspedes, e pode ser conduzida para uma pesquisa de qualidade em serviços. Gândara, Brea e Manosso (2013) confirmam dizendo que se faz necessário que os hotéis monitorem os sites com os depoimentos on-line de forma que possam melhorar os serviços oferecidos.

O próximo tópico apresenta a qualidade dos serviços na hotelaria, compreendendo os modelos de ferramentas utilizadas pelos pesquisadores que possibilitam fazer a análise dos Conteúdos Gerados pelos Usuários on-line, permitindo avaliar a qualidade dos serviços oferecidos pelos hotéis.

\section{QUALIDADE DOS SERVIÇOS NA HOTELARIA}

As empresas hoteleiras, influenciadas pela crescente exigência dos clientes e pela competitividade cada vez mais acirrada do setor, vêm trabalhando de forma intensa na melhoria da qualidade dos serviços oferecidos. Diferentemente do que acontece no consumo de outros produtos e serviços, os clientes de hotéis têm um contato sensorial com o local onde estão hospedados, sentindo-o, vivendo-o e interagindo com ele (CÂNDIDO; VIEIRA, 2003).

No tocante ao referencial teórico que orienta a produção científica sobre a qualidade em serviços turísticos de modo geral, Vasconcelos e Lezana (2014) 
selecionaram um portfólio bibliográfico publicado entre 2002 e 2012 e dele extraíram e analisaram as referências de maior destaque (Quadro 1).

\section{Quadro 1: Referências de maior destaque no portfólio}

\begin{tabular}{|c|c|c|}
\hline $\mathrm{N}^{\circ}$ & Autor (ano) & Referência \\
\hline$(1)$ & Oliver (1980) & $\begin{array}{l}\text { Oliver, R. L. A cognitive model of the antecedents and consequences of } \\
\text { satisfaction decisions. Journal of Marketing Research, 17(November), } \\
460-469,1980 .\end{array}$ \\
\hline$(2)$ & $\begin{array}{l}\text { Parasuraman, } \\
\text { Zeithaml \& Berry } \\
\qquad(1985)\end{array}$ & $\begin{array}{l}\text { Parasuraman, A.; Zeithaml, A.; Berry, L. L. A conceptual model of service } \\
\text { quality and implications for future search. Journal of Marketing, 49, 41- } \\
\text { 50, } 1985 \text {. }\end{array}$ \\
\hline (3) & $\begin{array}{l}\text { Parasuraman, } \\
\text { Zeithaml \& Berry } \\
\qquad(1988)\end{array}$ & $\begin{array}{c}\text { Parasuraman, A.; Zeithaml, A.; Berry, L. L. SERVQUAL: A multiple-item } \\
\text { scale for measuring consumer perceptions of service quality. Journal of } \\
\text { Retailing, 64(1), 12-40, } 1988 .\end{array}$ \\
\hline$(4)$ & Zeithaml (1988) & $\begin{array}{l}\text { Zeithaml, A. Consumer perceptions of price, quality, and value: a } \\
\text { means-end model and synthesis of evidence. The Journal of Marketing, } \\
52 \text { (July), 2-22, } 1988 .\end{array}$ \\
\hline$(5)$ & Grönroos (1990) & $\begin{array}{l}\text { Grönroos, C. Service management and marketing: managing the } \\
\text { moments of truth in service competition. Lexington-MA: Lexington } \\
\text { Books, } 1990 .\end{array}$ \\
\hline$(6)$ & Carman (1990) & $\begin{array}{l}\text { Carman, J. M. Consumer perceptions of service quality: An assessment } \\
\text { of the SERVQUAL dimensions. Journal of Retailing, 66(1), 35-55, } 1990 .\end{array}$ \\
\hline$(7)$ & Bolton \& Drew (1991) & $\begin{array}{l}\text { Bolton, R. N.; Drew, J. H. A multistage model of customers' assessments } \\
\text { of service quality and value. Journal of Consumer Research, 17(4), 375- } \\
\text { 384, } 1991 .\end{array}$ \\
\hline$(8)$ & Saleh \& Ryan (1991) & $\begin{array}{c}\text { Saleh, F.; Ryan, C. Analysing service quality in the hospitality industry } \\
\text { using the SERVQUAL model. Service Industries Journal, 11(3), 324-345, } \\
1991 .\end{array}$ \\
\hline$(9)$ & $\begin{array}{l}\text { Cronin Jr \& Taylor } \\
\text { (1992) }\end{array}$ & $\begin{array}{l}\text { Cronin Jr, J. J.; Taylor, S. A. Measuring service quality: a reexamination } \\
\text { and extension. The Journal of Marketing, 56(3), 55-68, } 1992 .\end{array}$ \\
\hline (10) & Hair et al. (1995) & $\begin{array}{l}\text { Hair, J. F.; et al. Multivariate data analysis. Englewood Cliffs-NJ: Prentice- } \\
\text { Hall, } 1995 .\end{array}$ \\
\hline (11) & $\begin{array}{l}\text { Zeithaml \& Bitner } \\
\text { (1996) }\end{array}$ & $\begin{array}{c}\text { Zeithaml, A.; Bitner, M. J. Services Marketing. London: McGraw-Hill, } \\
1996 .\end{array}$ \\
\hline$(12)$ & Oliver (1997) & $\begin{array}{l}\text { Oliver, R. L. Satisfaction: A behavioral perspective on the consumer. } \\
\text { New York: McGraw-Hill. Parasuraman, } 1997 .\end{array}$ \\
\hline (13) & $\begin{array}{l}\text { Cronin Jr, Brady \& } \\
\text { Hult (2000) }\end{array}$ & $\begin{array}{l}\text { Cronin Jr, J. J.; Brady, M. K.; Hult, G. T. M. Assessing the effects of quality, } \\
\text { value, and customer satisfaction on consumer behavioral intentions in } \\
\text { service environments. Journal of Retailing, 76(2), 193-218, } 2000 \text {. }\end{array}$ \\
\hline
\end{tabular}

Fonte: Adaptado de Vasconcelos e Lezana (2014)

Dentre os trabalhos apresentados, verifica-se apenas uma obra que trata do método de análise e tratamento de dados quantitativos (HAIR et al., 1995), outras 
duas sobre a gestão de operações de serviços (GRÖNROOS, 1990) e marketing de serviços (ZEITHAML; BITNER, 1996) e as demais tratam da qualidade em serviços (VASCONCELOS; LEZANA, 2014).

No âmbito da hotelaria, diversos estudos têm sido desenvolvidos para avaliar a qualidade dos serviços e a satisfação do consumidor com a aplicação de diferentes modelos de importância atestada e reconhecida. Dentre eles, destacam-se a utilização da escala SERVQUAL e o Modelo Gap, criados por Parasuraman, Zeithaml e Berry (1985; 1988), em que a satisfação do cliente é função da diferença entre expectativa e desempenho; e a escala SERVPERF, desenvolvida por Cronin e Taylor (1992), baseada somente na percepção de desempenho dos serviços. Meira (2012) destaca que a realização de pesquisas voltadas para a qualidade na prestação de serviços nos hotéis é de grande importância para o desenvolvimento do setor.

Na pesquisa realizada por Lee et al. (2004), examinou-se o grau de discrepância entre o desempenho esperado e percebido na qualidade do serviço em hotéis, tendo por base as cinco dimensões propostas nos modelos SERVQUAL e SERVPERF. Dentre os resultados obtidos, aferiu-se que o impacto da qualidade do serviço varia de acordo com a categoria do hotel. Essa variância também foi percebida no trabalho de Souza, Meira e Maske (2012), na aplicação do instrumento SERVQUAL em hotéis de uma, duas, três e quatro estrelas de Balneário Camboriú. Segundo Anjos e Abreu (2009), essas diferenças devem ser analisadas no momento em que são planejadas as estratégias de curto, médio e longo prazo dos hotéis.

Nos hotéis de cinco estrelas, de acordo com a pesquisa de Lee et al. (2004), a empatia aumentou a qualidade do serviço percebido. Por outro lado, em hotéis de categoria quatro estrelas, foi constatado que, além da empatia, a dimensão confiabilidade mostra-se fundamental. Já nos hotéis três estrelas, a qualidade do serviço foi influenciada pela empatia, capacidade de resposta e aspectos tangíveis, associados diretamente às instalações físicas e à aparência dos funcionários. Esta constatação, segundo os autores, sustenta o pressuposto de que a satisfação do cliente é influenciada pelas atitudes e comportamentos dos funcionários. 
Fazendo-se uso de um modelo adaptado da escala SERVQUAL, Anjos e Abreu (2009) compararam a expectativa e a percepção do cliente com a dos colaboradores de hotéis no tocante à qualidade dos serviços oferecidos. Os resultados da pesquisa indicaram a existência de diferentes pontos de vista entre as percepções dos clientes e dos colaboradores de hotéis, bem como entre as expectativas e as percepções dos clientes sobre os serviços oferecidos. Apontou-se também que, apesar da escala SERVQUAL se apresentar como uma ferramenta válida na mensuração da qualidade dos serviços, é necessário adaptá-la de acordo com o tipo de serviço prestado e as características específicas do público consumidor.

Lima-Filho, Marchiotti e Silva (2012) também fizeram uso da escala SERVQUAL para avaliar a satisfação dos consumidores em hotéis, nesse caso em Campo Grande (MS). Os resultados obtidos mostraram que, de modo geral, a rede hoteleira da cidade tem satisfeito seus clientes na maior parte das dimensões avaliadas. Os atributos determinantes para a satisfação dos consumidores foram a segurança e os equipamentos oferecidos pelas instalações, assim como cortesia, agilidade e eficiência dos funcionários no atendimento aos hóspedes.

Em publicação mais recente, Aquino, Jerônimo e Melo (2015), por meio de um estudo de caso e fazendo uso da escala SERVPERF, analisaram a percepção dos consumidores quanto aos serviços oferecidos em determinado hotel. Entre os resultados apontados, observou-se a necessidade de melhoria no atendimento, bem como na agilidade e na presteza dos colaboradores. Segundo os autores, a escala SERVPERF revelou-se como um instrumento útil no estudo. Afirmou-se, ainda, a importância da avaliação da qualidade num processo contínuo de melhoria no relacionamento entre os hotéis e seus clientes. Dessa maneira, Gândara, Brea e Manosso (2013) relatam que os empreendimentos hoteleiros devem olhar para a qualidade dos serviços, a satisfação dos clientes e a experiência para ganhar uma melhor reputação entre os consumidores. Por isso, a reputação on-line vem ganhando importância no âmbito do mercado hoteleiro, como forma de entender a percepção de qualidade e a satisfação do consumidor diante das experiências vividas por eles. 
A pesquisa realizada por Limberger et al .(2014) confirma esta tendência, pois buscou avaliar a satisfação dos serviços prestados em hotéis no Brasil de categorias 3, 4 e 5 estrelas utilizando o Conteúdo Gerado pelos Usuários no portal de viagens TripAdvisor, por meio do método de análise multivariada de correspondência múltipla.

Assim sendo, o presente artigo se propõe a utilizar o modelo SERFPERF como ferramenta para auxiliar os hotéis na análise do CGU, de forma que possam orientar suas estratégias na consolidação da sua posição no mercado, oferecendo serviços de melhor qualidade e mantendo os clientes satisfeitos, conforme será abordado no tópico seguinte.

\section{MODELO DE AVALIAÇÃO DA QUALIDADE DOS SERVIÇOS}

(SERVPERF)

Cronin e Taylor (1992) desenvolveram o modelo SERVPERF para medir a qualidade dos serviços como alternativa ao SERVQUAL, sendo que a principal diferença entre os dois instrumentos é que o SERVPERF considera apenas a percepção de desempenho (performance) do serviço. Nessa ótica, não são levadas em consideração as expectativas do consumidor, devido ao entendimento de que a avaliação por meio da percepção dos consumidores é a forma mais apropriada de mensuração da qualidade dos serviços prestados (CRONIN; TAYLOR, 1992; LEE et al., 2004; CAMISÓN; CRUZ; GONZÁLEZ, 2007; ANJOS; ABREU, 2009).

Com isso, o modelo SERVPERF compõe afirmativas sobre o desempenho do serviço, representando as cinco dimensões da qualidade desenvolvidas por Parasuraman, Zeithaml e Berry (1988), quais sejam: tangibilidade, confiabilidade, capacidade de resposta, segurança e empatia (CRONIN; TAYLOR, 1992; CAMISÓN; CRUZ; GONZÁLEZ, 2007).

A dimensão tangibilidade está ligada aos aspectos físicos e aos equipamentos. Já a confiabilidade representa se a empresa cumpre o que foi prometido no prazo determinado. A capacidade de resposta refere-se à disposição para auxiliar os clientes de forma responsável. Já a segurança está relacionada 
ao conhecimento, à cortesia e à capacidade de emitir confidencialidade. Por último, a empatia diz respeito à disposição em demonstrar interesse e atenção ao cliente (PARASURAMAN; ZEITHAML; BERRY, 1988).

Aquino, Jerônimo e Melo (2015) argumentam que realizar a avaliação de serviços é um processo difícil, pois cada cliente percebe um nível de qualidade, especificações e necessidades diferentes. Assim, ao fornecer um índice longitudinal das percepções da qualidade dos serviços, o modelo SERVPERF pode fornecer subsídios importantes na indicação de oportunidades de melhoria e apontar os pontos fortes e fracos dos hotéis (LEE et al., 2004), objeto de estudo deste artigo.

METODOLOGIA

Este trabalho consiste em um estudo qualitativo, de caráter exploratóriodescritivo (DENCKER, 2003), pois ao mesmo tempo em que objetiva analisar a percepção dos hóspedes de negócios sobre a qualidade dos serviços prestados pelos hotéis da cidade de Florianópolis, procura descrever suas respostas por meio das cinco dimensões da qualidade propostas no modelo SERVPERF (CRONIN; TAYLOR, 1992).

Inicialmente fez-se uma revisão bibliográfica acerca dos assuntos-chave que abordam a temática, por meio do acesso ao Portal de Periódicos da CAPES e da Biblioteca Digital Brasileira de Teses e Dissertações (BDTD). Para Dencker (2003), a pesquisa bibliográfica é desenvolvida com base em material já elaborado, a exemplo de livros e artigos científicos. Na sequência, para realizar o levantamento do conteúdo gerado pelo usuário, utilizou-se a técnica de pesquisa documental, que segundo Dencker (2003) consiste em documentos de primeira ou segunda mão conservados em arquivos de instituições públicas e privadas. É o caso deste estudo, que por meio de pesquisa no portal de viagens Booking.com, identificou os hotéis que continham avaliações de hóspedes de negócios, publicadas entre o período de 13 de maio de 2014 a 14 de julho de 2015, utilizando-se do Software NVIVO para análise de textos.

Faz-se necessário esclarecer aqui que há duas nomenclaturas utilizadas no referido site, uma incluindo os hóspedes em viagem a lazer e outra incluindo 
aqueles a negócios. Entretanto, para o desígnio desta pesquisa, foram verificadas somente as avaliações feitas pelos hóspedes de negócios. Já o Software NVIVO foi escolhido como suporte para análise dos dados, principalmente por disponibilizar diferentes funcionalidades que facilitam o processo de codificação e o agrupamento de textos.

Dos 344 hotéis publicados em Florianópolis, 249 foram avaliados por hóspedes de negócios, totalizando 3.523 avaliações. Para fins desta pesquisa, foram escolhidos os 06 (seis) hotéis com maior número de comentários, que juntos somaram 2.287 postagens. Dessa quantidade, contatou-se que somente 1.133 possuíam texto com a percepção dos hóspedes sobre os serviços prestados pelo hotel. Nas demais avaliações, a empresa Booking.com informa que não consta qualquer tipo de informação dos hóspedes, além da nota concedida pelos mesmos. Por conseguinte, a análise deste trabalho se limita ao número de avaliações com texto (Quadro 2).

Quadro 2: Avaliações geradas pelo usuário nos 06 hotéis de Florianópolis

\begin{tabular}{|c|c|c|c|}
\hline Hotel & $\begin{array}{c}\text { Classificação do hotel } \\
\text { Booking.com }\end{array}$ & Total de avaliações & $\begin{array}{c}\mathbf{N}^{\circ} \text { de avaliações com } \\
\text { texto }\end{array}$ \\
\hline Cecomtur & 3 estrelas & 560 & 291 \\
\hline Castelmar & 3 estrelas & 364 & 189 \\
\hline Florianópolis Palace & 4 estrelas & 318 & 180 \\
\hline Valerim Center & 2 estrelas & 367 & 170 \\
\hline Porto da Ilha & 4 estrelas & 342 & 154 \\
\hline Valerim Plaza & 3 estrelas & 336 & 149 \\
\hline
\end{tabular}

Fonte: Booking.com (elaborado pelos autores).

A amostra representativa foi constituída por 06 (seis) hotéis que serão tratados como Alpha, Betta, Delta, Gamma, Epsilon e Zeta nos resultados do estudo. Na apresentação dos resultados, o estudo analisará os dados de forma genérica, sem realizar comparações entre os hotéis.

A Booking.com se destaca como uma das empresas líderes em informação relacionada À hospedagem no mundo, assim como no Brasil. Segundo pesquisa realizada no mês de dezembro de 2012, este foi o site de viagens brasileiro mais visitado na categoria Destinos e Acomodação, representando 15,7\% dos acessos (PANROTAS, 2013). A Booking.com já foi objeto de alguns estudos, a 
exemplo de Mendes-Filho e Carvalho (2014); Gândara, Brea e Manosso (2013), citados neste trabalho.

A empresa foi fundada em 1996, com sede em Amsterdã, na Holanda e desde então tem se expandido. Atualmente opera em 63 países, com 165 escritórios, e oferece mais de 694.103 propriedades em 215 diferentes países (BOOKING, on-line, 2015).

Neste portal, somente hóspedes com reserva e hospedagem realizada por intermédio da Booking.com podem avaliar os serviços prestados em sua estadia. Após o check out o usuário é convidado, através do envio de um e-mail, a fazer sua avaliação. Esse procedimento reforça, de certa forma, a credibilidade nas avaliações publicadas (BOOKING, on-line, 2015).

Para mensurar o desempenho percebido pelos hóspedes por meio do Conteúdo Gerado pelo Usuário (CGU) no Booking.com, utilizou-se das dimensões do modelo SERVPERF de Cronin e Taylor (1992). Assim, os comentários foram codificados no Software NVIVO, colocando-os em ordem de prioridade de acordo com cada dimensão do modelo proposto. A disposição das informações permitiu realizar a análise qualitativa, identificando os pontos fortes e fracos que possuem maior incidência no relato dos hóspedes, e que podem servir de base para a tomada de decisão das empresas hoteleiras, orientando suas estratégias para a melhoria dos seus serviços.

\section{RESULTADOS E DISCUSSÕES}

Os resultados desta pesquisa serão apresentados dentro das cinco dimensões da escala SERVPERF de Cronin \& Taylor (1992), quais sejam: tangibilidade, confiabilidade, capacidade de resposta, segurança e empatia. Foram analisados os comentários publicados no site de viagens Booking.com a partir dos elementos de maior destaque apontados pelos hóspedes de negócios nos seis hotéis de Florianópolis, relacionando-os com as dimensões da qualidade do referido modelo e aferindo-Ihes graus de prioridade. 
Quadro 3: Dimensões da qualidade $X$ Pontos Fortes/Pontos Fracos identificados pelos hóspedes

\begin{tabular}{|c|c|c|c|c|}
\hline Dimensão & Pontos Fortes & $\begin{array}{c}\text { Grau } \\
\text { Prioridade }\end{array}$ & Pontos Fracos & $\begin{array}{c}\text { Grau } \\
\text { Prioridade }\end{array}$ \\
\hline \multirow{5}{*}{ Tangibilidade } & Localização & 3 & $\begin{array}{c}\text { Equipamentos e instalações em } \\
\text { mau estado de conservação/ } \\
\text { antigos }\end{array}$ & 3 \\
\hline & $\begin{array}{l}\text { Qualidade e } \\
\text { variedade dos itens } \\
\text { do café da manhã }\end{array}$ & 3 & $\begin{array}{l}\text { Acesso à internet wi-fi ruim/ não } \\
\text { funcionou }\end{array}$ & 2 \\
\hline & $\begin{array}{c}\text { Conforto/ espaço do } \\
\text { quarto }\end{array}$ & 3 & Limpeza do hotel/ acomodações & 1 \\
\hline & Limpeza & 2 & Ruído/ isolamento acústico & 1 \\
\hline & Ambiente moderno & 1 & Estacionamento (valor, tamanho) & 1 \\
\hline \multirow{4}{*}{ Confiabilidade } & \multirow{4}{*}{$\begin{array}{l}\text { Relação custo- } \\
\text { benefício }\end{array}$} & \multirow{4}{*}{2} & $\begin{array}{l}\text { Confiabilidade dos valores } \\
\text { aplicados no fechamento da conta }\end{array}$ & 1 \\
\hline & & & Clareza da informação prestada & 1 \\
\hline & & & $\begin{array}{l}\text { Qualidade/funcionamento das } \\
\text { áreas de lazer }\end{array}$ & 1 \\
\hline & & & $\begin{array}{l}\text { Imagens das acomodações } \\
\text { veiculadas não condizem com a } \\
\text { realidade }\end{array}$ & 1 \\
\hline \multirow{2}{*}{$\begin{array}{l}\text { Capacidade de } \\
\text { resposta }\end{array}$} & $\begin{array}{l}\text { Rapidez/agilidade } \\
\text { no atendimento } \\
\text { prestado }\end{array}$ & 3 & $\begin{array}{l}\text { Rapidez/agilidade no atendimento } \\
\text { prestado }\end{array}$ & 1 \\
\hline & $\begin{array}{l}\text { Equipe prestativa/ } \\
\text { disponível para } \\
\text { ajudar }\end{array}$ & 2 & $\begin{array}{l}\text { Equipe prestativa/disponível para } \\
\text { ajudar }\end{array}$ & 1 \\
\hline \multirow{3}{*}{ Segurança } & \multirow{3}{*}{$\begin{array}{l}\text { Colaboradores } \\
\text { capacitados/ } \\
\text { treinados }\end{array}$} & \multirow{3}{*}{1} & Estado de conservação do hotel & 1 \\
\hline & & & $\begin{array}{c}\text { Competência para resolver } \\
\text { problemas }\end{array}$ & 1 \\
\hline & & & Segurança no entorno do hotel & 1 \\
\hline \multirow[b]{2}{*}{ Empatia } & \multirow{2}{*}{$\begin{array}{l}\text { Hospitalidade dos } \\
\text { colaboradores da } \\
\text { recepção }\end{array}$} & \multirow[b]{2}{*}{1} & Pouca flexibilidade de horários & 1 \\
\hline & & & $\begin{array}{c}\text { Falta de colaboradores suficientes } \\
\text { no check in/out para atender a } \\
\text { necessidade dos hóspedes }\end{array}$ & 1 \\
\hline
\end{tabular}

Fonte: Booking.com (elaborado pelos autores)

Os pontos fortes e pontos fracos identificados pelos hóspedes foram alocados de acordo com o seu grau de prioridade, sendo 3 - muito importante, 2 - importância moderada e 1 - pouco importante. O grau de prioridade foi aferido conforme o número total de ocorrências apontadas pelos hóspedes nos hotéis pesquisados. 
No total constaram 130 registros considerados de pouca importância, 255 com importância moderada e 515 apontadas como muito importante.

A partir da análise do Quadro 3, pode-se perceber que, dentro da dimensão Tangibilidade, os principais aspectos positivos observados pelos hóspedes, com prioridade muito importante, foram: a localização próxima ao local de trabalho ou evento que o hóspede participou; o café da manhã, com diversidade e reposição dos alimentos; e o conforto/espaço do quarto, permitindo um bom descanso. Com prioridade moderada, os hóspedes apontaram a limpeza das instalações e deram pouca importância no que se refere à modernidade do ambiente.

Com base nos comentários publicados, verifica-se que a localização do hotel é o principal fator levado em consideração na escolha da hospedagem pelos hóspedes de negócios. Essa constatação corrobora com o resultado obtido na pesquisa realizada pelo Convention \& Visitors Bureaux de Florianópolis em parceria com a Fundação Getúlio Vargas (2010), que verificou a preferência dos pesquisados por hotéis localizados na região central da cidade, devido à proximidade dos equipamentos turísticos e espaços para eventos. Os hóspedes dos hotéis Zeta e Delta afirmaram em suas avaliações, respectivamente, que:

A localização é excelente para quem está na cidade a trabalho. 0 hotel fica próximo a várias empresas e órgãos públicos.

O hotel está localizado no centro comercial de Florianópolis. Há diversos bancos, restaurantes e lojas comerciais próximo. A rodoviária de Florianópolis é muito perto, cinco minutos de caminhada.

Quanto aos pontos fracos encontrados nesta dimensão, verificam-se com maior grau de importância os equipamentos e as instalações, que se encontram em mau estado de conservação ou são muito antigos, a exemplo dos elevadores; com moderada importância os hóspedes assinalaram a qualidade do acesso à internet wi-fi, que muitas vezes não funciona; e com pouca importância apontaram a limpeza, que apesar de constar em menor número de avaliações negativas do que positivas, contribui para a percepção negativa da qualidade dos serviços; o ruído/isolamento acústico das acomodações, prejudicando a qualidade do sono; bem como a cobrança/ tamanho da garagem oferecida pelos hotéis. Na ótica do hóspede do hotel Betta: 
Barulho excessivo do pessoal da limpeza no apartamento do andar superior às 8:30 da manhã de domingo. Ar condicionado sujo, mofado, antigo e muito barulhento. Tive que desligá-lo para conseguir dormir. Wi-Fi só caía. Elevador demorado e precisa de mais manutenção.

Na dimensão Confiabilidade, quanto aos pontos fortes os hóspedes identificaram com moderado grau de importância aspectos relacionados ao custo-benefício da hospedagem, que de forma geral respondeu à necessidade dos clientes. Por outro lado, os pontos fracos com pouca importância foram: a confiabilidade dos valores aplicados no fechamento da conta, com cobranças equivocadas percebidas pelos clientes; a clareza das informações prestadas ao hóspede, para evitar maiores transtornos e dificuldades; a qualidade e o funcionamento das áreas de lazer, por apresentarem-se inoperantes em diversas situações; bem como as imagens das acomodações veiculadas não condizerem com a acomodação obtida durante a estadia, gerando insatisfação relacionada ao cumprimento das promessas realizadas. O relato do hóspede do hotel Zeta confirma o ponto fraco desta dimensão,

A oscilação nos preços das diárias. Fiz a reserva com mais de um mês de antecedência e paguei um valor bem acima daqueles que fizeram mais perto da viagem. Conversei com o hotel, mas mesmo assim foram inflexíveis com a alteração de valor.

No que se refere à dimensão Capacidade de Resposta, evidenciaram-se os mesmos aspectos tanto na percepção positiva quanto negativa dos hóspedes. Contudo, faz-se necessário observar que houve maior grau de importância dos hóspedes à rapidez/agilidade no atendimento dos colaboradores, e em moderada importância à hospitalidade/disponibilidade da equipe em assistir ao hóspede, no que tange aos pontos fortes da prestação dos serviços pelos hotéis. Já os pontos fracos apareceram com pouca importância. Na visão do hóspede do hotel Gamma:

O atendimento, principalmente do pessoal da recepção, foi muito bom. Já é segunda vez que me hospedo neste hotel e só tenho elogios para sua equipe de atendimento.

Por sua vez, na dimensão Segurança, verificou-se a percepção positiva dos hóspedes em grau de pouca importância em relação à capacidade/treinamento 
dos colaboradores para o desempenho das suas funções. Entretanto, como pontos fracos também com pouca importância apontada pelos hóspedes foram destacados: o estado de conservação dos hotéis, que apresentam estruturas antigas e precisam passar por uma reforma; a competência para resolver problemas, que geram insatisfação nos serviços prestados; tal como a segurança no entorno do hotel. Como descreve o hóspede do hotel Alpha:

Tem muita escada e não tem rampa para as pessoas com deficiência
e nem para o transporte das bagagens. Os elevadores são pequenos
e estão sempre em manutenção. Tem uma barreira ao descer da
escada para o atendimento das pessoas no balcão que está de tal
modo posicionado que pode causar acidentes tendo em vista existir
duas escadas entre os elevadores e a saída na rua.

Finalmente, na dimensão Empatia, constatou-se pouca importância na avaliação dos hóspedes, tanto nos pontos fortes quanto nos pontos fracos. No que se refere aos aspectos fortes, os clientes estão satisfeitos com a hospitalidade e a gentileza dos colaboradores da recepção. De acordo com o relato do hóspede do hotel Epsilon:

Adorei o atendimento de todos os funcionários do hotel! Todos muito prestativos, educados, eficientes e organizados!

No entanto, a falta de colaboradores suficientes no check in e check out compromete o atendimento mais individualizado ao hóspede. Além disso, evidenciou-se que a inflexibilidade dos hotéis no tocante aos horários de entrada e saída geram constrangimentos e insatisfação aos clientes. Conforme descreve um hóspede do hotel Alpha e Epsilon respectivamente:

Falta de flexibilidade no check in e no check out, não deixam em hipótese alguma entrar uns minutinhos antes das 14:00 ou sair uns minutinhos depois, nem mudar o quarto.

Cheguei por volta das 13:30 e tive que aguardar até as 14:10 para poder entrar uma vez que tinha feito a reserva, outra coisa o horário da diária segundo informações passadas seria das 14:00 as 12:00, é a primeira vez que vejo isso então não é uma diária?

Tendo em vista os resultados da pesquisa, cabe destacar que a dimensão tangibilidade teve o maior grau de prioridade nas avaliações dos hóspedes. Com 
isso demonstra-se a necessidade dos hotéis adequarem as evidências físicas assinaladas, para aumentar a percepção dos clientes em relação à qualidade dos serviços prestados.

\section{CONSIDERAÇÕES FINAIS}

A hotelaria voltada ao segmento de negócios e eventos no município de Florianópolis encontra-se em expansão da sua oferta, na medida em que o atendimento a esse perfil de cliente permite reduzir os efeitos da sazonalidade receados pelos hotéis no período fora da temporada de verão.

Um fator propulsor dessa expansão caracteriza-se pela consolidação da região metropolitana da grande Florianópolis, que se destaca no cenário nacional como polo tecnológico, além da existência de um diversificado parque industrial e uma oferta variada de serviços. Consequentemente, verifica-se a existência de uma demanda latente voltada para o turismo de negócios e eventos na cidade.

Neste sentido, o estudo atingiu o objetivo proposto de analisar a percepção dos hóspedes de negócios em relação à qualidade dos serviços prestados pelos seis hotéis de Florianópolis, com maior número de comentários de viajantes de negócios, possibilitando retratar assim os aspectos considerados mais significativos por essa demanda de clientes.

No que se refere à tangilidade, pode-se destacar que um dos aspectos positivos na percepção dos hóspedes foi a localização próxima ao local de trabalho ou evento no qual participaram. Isso se deve, principalmente, ao fato de os hotéis estarem localizados na região central do município, que corrobora com a proximidade dos equipamentos turísticos e dos espaços para eventos.

Destaca-se quanto à confiabilidade, que os hóspedes levam em consideração principalmente o aspecto custo-benefício, sendo que de maneira geral os hotéis pesquisados supriram suas necessidades. Todavia, nota-se que alguns aspectos fundamentais da qualidade dos serviços prestados pelos hotéis deixaram a desejar nesta dimensão, como a falta de clareza nas informações dadas, a não operacionalização de todos os espaços de lazer oferecidos pelos hotéis, assim 
como a não correspondência, em muitos casos, da acomodação veiculada na mídia com aquela obtida durante a sua hospedagem. Sugere-se a revisão destes pontos pelos hotéis, para que possam aprimorar seus serviços, buscando maior qualidade e satisfação na experiência dos hóspedes.

Referente à capacidade de resposta do hotel com seus clientes, evidenciou-se que houve, no geral, rapidez no atendimento e na prestatividade da equipe.

Quanto à segurança, os hóspedes se mostraram satisfeitos sobretudo em relação à capacidade dos colaboradores em desempenhar suas funções. Contudo, tratando-se de aspectos como o estado de conservação dos hotéis, a competência para resolver problemas e a segurança no entorno do hotel, os comentários apontaram deficiências a serem resolvidas.

No que diz respeito à empatia, foram destacados o número insuficiente de colaboradores no momento do check in e do check out e a inflexibilidade dos hotéis com relação aos horários de entrada e saída, o que pode ser aprimorado para que sua experiência seja mais positiva.

A utilização do modelo SERVPERF neste trabalho mostrou-se satisfatória, pois conforme mencionado por Lee et. al (2004), fornece bases para indicar oportunidades de melhoria e apontar os pontos fortes e fracos dos hotéis. Por meio do Conteúdo Gerado pelo Usuário no site de viagens Booking.com e com auxílio do Software NVIVO, foi possível organizá-los em grau de prioridade, conforme o número de ocorrências dos elementos verificados.

O estudo avaliou aspectos internos dos serviços prestados, ou seja, serviços que estão exclusivamente no controle da empresa. Portanto, os resultados poderão auxiliar os hotéis na formulação das suas estratégias, com a finalidade de produzir ações que busquem a melhoria contínua e o diferencial competitivo frente às necessidades dos hóspedes de negócios.

Quanto às limitações da pesquisa, há de ser considerada a dificuldade em mensurar quantitativamente a qualidade do serviço oferecido pelos hotéis. Este fato levou os autores a optarem pelo estudo dos pontos fortes e fracos percebidos pelo hóspede ao utilizar seus serviços. Igualmente, destaca-se a incapacidade de traçar o perfil dos respondentes através do site de viagens 
Booking.com, o dificulta uma maior compreensão dos pesquisadores sobre a autonomia destes hóspedes na escolha do seu hotel.

Por fim, aponta-se como outra limitação a ser considerada o caráter não probabilístico da amostragem. Deste modo, sugere-se para trabalhos futuros a reaplicação do estudo em outras localidades para que se possa fazer um comparativo entre hotéis da mesma categoria e/ou o emprego de novos instrumentos para tratamento dos dados.

\section{REFERÊNCIAS}

$A B E O C$. Turismo de eventos gera discussões e investimentos em Florianópolis (2015). Disponível em: http://www.abeoc.org.br/2015/03/turismo-de-eventos-gera-discussoes-einvestimentos-em-florianopolis/>. Acesso em 07 abr. 2015.

ANJOS, S. J. G. dos.; ABREU, A. F. de. La medición de la calidad de servicio: una aplicación en empresas hoteleras. Revista europea de dirección y economía de la empresa, v. 18, n. 2, p. 175-186, 2009.

AQUINO, J. T. de.; JERÔNIMO, T. de. B.; MELO, F. J. C. de. Avaliação da qualidade em serviço de um hotel pelo método servperf. Revista Pensamento Contemporâneo em Administração, v. 9, n. 1, p. 127-144, 2015.

BOOKING. Portal. Disponível em: <http://www.booking.com>. Acesso em: 09 jul. 2015.

CAMISÓN, C.; CRUZ, S.; GONZÁLEZ, T. Gestión de la Calidad: Conceptos, Enfoques, Modelos y Sistemas. Madrid: Pearson Educación, 2007.

CÂNDIDO, I.; VIEIRA, E. V. Gestão de hotéis: técnicas, operações e serviços. Caxias do Sul: EDUCS, 2003.

Convention \& Visitors Bureaux Florianópolis e Região; Fundação Getúlio Vargas. Estudo de perfil e impacto econômico dos eventos nacionais e internacionais realizados em Florianópolis-SC. Disponível em: <http://floripaconvention.com.br/index.asp?dep $=114 \& \mathrm{c}$ ategoria $=10 \&$ associado=57>. Acesso em 08 abr. 2015.

CORRÊA, C.;HANSEN, D. R.. Qualidade deserviços em restaurantes deSão Paulo premiados pelo TripAdvisor: análise do conteúdo gerado pelo usuário. Revista Hospitalidade, p. 271290, 2015. 
CRONIN, J. J.; TAYLOR, S. A. Measuring service quality: a reexamination and extension. Journal of Marketing, vol. 56, no 03, p. 55-68, 1992.

CRONIN, J.; TAYLOR, S. Servperf versus Servqual: reconciling performance based and perceptions minus expectations measurement of service quality. Journal of Marketing, $\mathrm{v}$. 58, n. 1, p. 125-131, 1994.

DENCKER, A. Métodos e técnicas de pesquisa em turismo. São Paulo: Futura, 2003.

FERREIRA, L. B. Estratégias de segmentação da hotelaria para o turismo de negócios: um estudo em São Luís (MA). 2010. Tese de Doutorado. Universidade de São Paulo.

FRIEDLANDER, R. J. Tendencias en la gestión de la reputación y las opiniones online de hoteles (2012). Disponível em: <http://www.hosteltur.com/185350_tendencias-gestionreputacion-opiniones-online-hoteles.html>. Acesso em: 18 jun. 2015.

GÂNDARA, José Manoel Gonçalves; FRAIZ BREA, Jose Antonio; MANOSSO, Franciele Cristina. Calidad de la experiencia en los hoteles termales de Galicia, España: Un análisis a través de la reputación online. Estudios y perspectivas en turismo, v. 22, n. 3, p. 492-525, 2013.

GRÖNROOS, C. A service quality model and its marketing implications. European Journal of Marketing. v. 18, n. 4, p. 36-44, 1984.

LEE, Yong-Ki et al. Exploring the role of service value in the relationship between service quality and customer satisfaction. International journal of hospitality \& tourism administration, v. 5, n. 1, p. 67-86, 2004.

LIMA-FILHO, D. de. O.; MARCHIOTTI, I. Z.; SILVA, F. Q. Expectativas versus Satisfação dos Consumidores da Rede Hoteleira de Campo Grande-MS, Brasil. Revista Turismo em Análise, v. 23, n. 1, p. 54-77, 2012.

LIMBERGER, P. F; ANJOS, F. A; MEIRA, J. V. S; ANJOS, S. J. G. Satisfaction in hospitality on TripAdvisor.com: An analysis of the correlation between evaluation criteria and overall satisfaction. Tourism \& Management Studies, v. 10, n. 1, p. 59-65, 2014.

MARCHIORI, E.; CANTONI, L. The online reputation construct: Does it matter for the tourism domain? A literature review on destinations' online reputation. Information Technology \& Tourism, v. 13, n. 3, p. 139-159, 2011.

MEIRA, J. V. de. S. A qualidade dos serviços na hotelaria brasileira: uma avaliação das redes hoteleiras sob a ótica de seus gestores. 2012. 193f. Dissertação (Mestrado em Turismo e Hotelaria) - Universidade do Vale do Itajaí, Balneário Camboriú, Santa Catarina, 2012. 
MENDES-FILHO, L.; CARVALHO, M. S. D. De. Factores que influyen en el uso del contenido generado por el usuário en internet. Un estúdio preliminar con viajeros brasileños. Estudios y Perspectivas en Turismo, v. 23, p. 607-625, 2014.

MOENS, M. F.; LI, J.; CHUA, T. S. Mining user generated content. Chapman and Hall/CRC, 2014.

MTUR. Pesquisa Anual de Conjuntura Econômica do Turismo $1^{\text {a }}$ edição (2015). Disponível em: <http://www.dadosefatos.turismo.gov.br/dadosefatos/conjuntura economica/pesquisa_conjuntura_turismo/. Acesso em: 26 fev. 2016.

MTUR. Turismo de negócios e eventos: orientações básicas. 2 ed. Brasília: Ministério do Turismo, 2010.

MTUR. Ministro destaca a importância da internet para o crescimento do turismo. Disponível em: <http://www.turismo.gov.br/turismo/noticias/todas_noticias/20141125_5. html>. Acesso em: 17 jun. 2015.

PANROTAS. Sites da Decolar, Booking e Tam são os mais visitados, 2013. Disponível em: <http://www.panrotas.com.br/noticia-turismo/mercado/sites-da-decolar-booking-e-tamsao-os-mais-visitados_84472.html?pesquisa=1 >. Acesso em: 09 jul. 2015.

PARASURAMAN, A.; V. ZEITHAML; L. BERRY. A conceptual model of service quality and its implication for future research. Journal of Marketing, v. 49, p. 41-50, 1985.

Servqual: A multiple-item scale for measuring consumer perceptions of service quality. Journal of Retailing, v. 64, n. 1, p. 12-40, 1988.

PHOCUSWRIGHT. The Phocuswright Yearbook 2014: The Year Ahead in Digital Travel. Disponível em: http://www.phocuswright.com/Travel-Research/Market-Overview-Sizing/ThePhocuswright-Yearbook-2014-The-Year-Ahead-in-Digital-Travel. Acesso em 20 fev. 2016.

PINE II, B. J.; GILMORE, J. H. The experience economy: work is theatre and every business a stage. Boston: Harvard Business School Press, 1999.

QUIROGA, R. M.; MONDO, T. S.; DE CASTRO JÚNIOR, D. F. L. Reputação online como instrumento para melhoria de serviços: um estudo na hotelaria de Garopaba e ImbitubaSanta Catarina. Revista de Turismo Contemporâneo, v. 2, n. 1, 2014.

SARTORI, R. V.; REIS, D. R. Gerenciamento da Reputação Digital como ferramenta de apoio a gestão da inovação. XXX Encontro Nacional de Engenharia de Produção. Maturidade e desafios da Engenharia de Produção: competitividade das empresas, condições de trabalho, meio ambiente. São Carlos, SP, 2010. 
SILVA, D. S. da.; MENDES-FILHO, L. Uma análise preliminar do uso de comentários na internet na escolha de um destino de viagem. Revista Turismo: estudos e práticas, v. 2, n. 2, 2014.

SOUZA, E. C.; MEIRA, J. V. de. S.; MASKE, D. C. A medição da qualidade dos serviços prestados em hotéis de Balneário Camboriú, SC: uma aplicação do modelo SERVQUAL. Rosa dos Ventos -Turismo e Hospitalidade, v. 4, n. 4, 2012.

VASCONCELOS, A. M.; LEZANA, Á. G. R. Referencial teórico que orienta a produção científica qualificada sobre a qualidade em serviços turísticos. Tourism \& Management Studies, v. 10, n. 2, p. 133-137, 2014.

YACOUEL, N; FLEISCHER, A. The role of cybermediaries in reputation building and price premiums in the online hotel market. Journal of Travel Research, v. 51, n. 2, p. 219-226, 2012.

YAMAMOTO, H; ISHIDA, $\mathrm{K}$; OHTA, T. Modeling Reputation Management System on Online C2C Market. Computational \& Mathematical Organization Theory, v. 10, p. 165178, 2004. 